\title{
The use of the elevated plus maze as an assay of anxiety-related behavior in rodents
}

\author{
Alicia A Walf ${ }^{1} \&$ Cheryl A Frye ${ }^{1-4}$ \\ ${ }^{1}$ Department of Psychology, ${ }^{2}$ Department of Biological Sciences and ${ }^{3}$ The Centers for Neuroscience and ${ }^{4}$ Life Sciences Research, The University at Albany-SUNY, \\ Albany, New York, USA. Correspondence should be addressed to C.A.F. (cafrye@albany.edu, aawalf@yahoo.com). \\ Published online 1 March 2007; doi:10.1038/nprot.2007.44

\begin{abstract}
The elevated plus maze is a widely used behavioral assay for rodents and it has been validated to assess the anti-anxiety effects of pharmacological agents and steroid hormones, and to define brain regions and mechanisms underlying anxiety-related behavior. Briefly, rats or mice are placed at the junction of the four arms of the maze, facing an open arm, and entries/duration in each arm are recorded by a video-tracking system and observer simultaneously for $5 \mathrm{~min}$. 0ther ethological parameters (i.e., rears, head dips and stretchedattend postures) can also be observed. An increase in open arm activity (duration and/or entries) reflects anti-anxiety behavior. In our
\end{abstract} \\ laboratory, rats or mice are exposed to the plus maze on one occasion; thus, results can be obtained in 5 min per rodent.
}

\section{INTRODUCTION}

The elevated plus maze has been described as a simple method for assessing anxiety responses of rodents by File and co-workers ${ }^{1}$. A task, using a Y-shaped apparatus that included an elevated open alley, which produced a strong approach-avoidance conflict, and an enclosed alley, which did not, was first described by Montgomery ${ }^{2}$. This task was modified into an elevated maze with four arms (two open and two enclosed) that are arranged to form a plus shape and was described by Handley and Mithani ${ }^{3}$. These authors described the assessment of anxiety behavior of rodents by using the ratio of time spent on the open arms to the time spent on the closed arms. Unlike other behavioral assays used to assess anxiety responses that rely upon the presentation of noxious stimuli (i.e., electric shock, food/water deprivation, loud noises, exposure to predator odor, etc.) that typically produce a conditioned response, the elevated plus maze relies upon rodents' proclivity toward dark, enclosed spaces (approach) and an unconditioned fear of heights/open spaces (avoidance) $)^{4}$.

There is great diversity in possible applications of the elevated plus maze. To name a few, prescreening of newly developed pharmacological agents for treatment of anxiety-related disorders can be carried out. The anxiolytic and anxiogenic effects of pharmacological agents, drugs of abuse and hormones can be investigated. The effects of reproductive senescence/aging and/or pre-, peri- or postnatal exposure to various stressors can be assessed. Furthermore, beyond its utility as a model to detect anxiolytic effects of benzodiazepine-related compounds, the elevated plus maze can be used as a behavioral assay to study the brain sites (e.g., limbic regions, hippocampus, amygdala, dorsal raphe nucleus, etc. ${ }^{5,6}$ ) and mechanisms (e.g., GABA, glutamate, serotonin, hypothalamic-pituitary-adrenal axis neuromodulators, etc. ${ }^{1,3,7-12}$ ) underlying anxiety behavior. Indeed, the elevated plus maze has been used as a model of state, unconditioned anxiety for over two decades, and there are now over 2,000 papers related to this topic. Because a lengthy discussion of these key findings is beyond the scope of this protocol, readers are referred to several excellent and instructive reviews ${ }^{13-21}$ ).

Behavioral responses in the elevated plus maze are easily assessed and quantified by an observer. Briefly, rodents are placed in the intersection of the four arms of the elevated plus maze and their behavior is typically recorded for $5 \mathrm{~min}$. This was based upon the early studies by Montgomery ${ }^{2}$ that revealed that rats demonstrated the most robust avoidance responses in the first $5 \mathrm{~min}$ after placement in the elevated open alleys. The behaviors that are typically recorded when rodents are in the elevated plus maze are the time spent and entries made on the open and closed arms. Behavior in this task (i.e., activity in the open arms) reflects a conflict between the rodent's preference for protected areas (e.g., closed arms) and their innate motivation to explore novel environments. Anti-anxiety behavior (increased open arm time and/or open arm entries) can be determined simultaneously with a measure of spontaneous motor activity (total and/or closed arm entries), albeit the arm entries made in the maze may not be an optimal measure of motor activity. Other ethological measures that can be observed in rodents in the maze are the number of rears, head dips, fecal boli, freezing or stretched-attend postures.

Face validity of the elevated plus maze

The elevated plus maze has face validity, which is the ability of a task to appear to measure what it is supposed to measure. For instance, in the elevated plus maze, the anxiety or fear of open spaces/heights of rodents seems to be measured. In this task, the open arms are avoided and rodents spend the majority of the time in this task in the closed arms of the maze. Other anxiety-related behaviors of rodents, such as freezing/immobility and defecation, are increased on the open arms of the maze compared to the closed $a r m s^{1}$.

Construct validity of the elevated plus maze

The elevated plus maze has construct validity. Construct validity refers to whether an observable dependent variable, such as time spent in the open arms of the elevated plus maze, used measures an unobservable construct, such as anxiety. This is demonstrated by anxiogenic drugs reducing time spent on the open arms and anxiolytic drugs increasing the time spent on the open arms of the elevated plus maze ${ }^{1}$.

Predictive validity of the elevated plus maze The elevated plus maze has predictive validity, which is defined as the extent to which the dependent measure predicts behavior on a 
related measure. We have shown that increased open arm activity occurs in rodents that also demonstrate increased central square entries in a brightly lit open field ${ }^{22}$. Furthermore, plasma corticosterone is increased with open arm exposure and is positively correlated with risk assessment behavior (i.e., stretchattend postures) in the elevated plus maze ${ }^{23,24}$.

\section{Effects of novel environment pre-exposure}

Pre-exposure to a different novel environment, such as an open field or hole-board task, has been used in studies assessing anxiety behavior of rodents. In some studies, exposure to a novel environment immediately before testing in the elevated plus maze increases motor activity in the elevated plus maze and a greater likelihood of entering the open arms of the maze $e^{1,25,26}$. Although indices of anxiety behavior in the elevated plus maze do not correlate with the amount of exploration in a hole-board ${ }^{27}$, using the hole-board task immediately before elevated plus maze testing can provide additional indices of activity and exploration (i.e., rearing and head dipping ${ }^{25,26}$, which are independent of plus maze exposure, to rule out changes in open arm exploration being due to changes in general activity and/or exploratory motivation. For instance, this method has been successfully used to demonstrate anxioselectivity of the long-lasting impact of experimental epilepsy on rodents and the impact of predator stress exposure (both experimental situations that can alter activity) on plus maze anxiety ${ }^{28,29}$.

In our laboratory, rats or mice are often run through a battery of tasks, which include testing in the open field followed by the elevated plus maze and then subsequent assessment in other measures of affect (i.e., the forced swim test) and/or social and/or sexual behavior (social interaction, propinquity and/or paced mating). It is also common in our laboratory to run each task on separate occasions. By comparing across studies in our laboratory, we have found little evidence to suggest differences in elevated plus maze behavior of rats or mice that are tested in a battery of behavioral tasks that includes the elevated plus maze versus testing in the elevated plus maze and no other tasks on a separate occasion. Although this suggests that, in our laboratory, pre-exposure to another testing environment does not alter subsequent behavior of rats or mice in the elevated plus maze, careful consideration of this should be made when designing experiments using the elevated plus maze.

\section{Utility of a single test session in the elevated plus maze}

When assessing the anxiolytic properties of pharmacological agents, many investigators use pre-exposure to the elevated plus maze. Although some early reports did not suggest that prior exposure to the elevated plus maze produced test decay and altered subsequent responding in the task ${ }^{1,8,30}$, recent reports suggest that there is some evidence of test decay effects, that is, there are differences in elevated plus maze behavior when rodents are exposed to the plus maze on more than one occasion. For instance, decreased activity on the open arms of the maze is typical on the second exposure to this task compared to the first exposure ${ }^{31-37}$. Given these effects, our laboratory and others have used the elevated plus maze in a slightly different way by using a single testing session and assessing behavior as a response to this novel situation that produces unconditioned avoidance responses toward the open arms. Indeed, this approach is now what is typically used. It must be noted that, although retesting in the plus maze can be

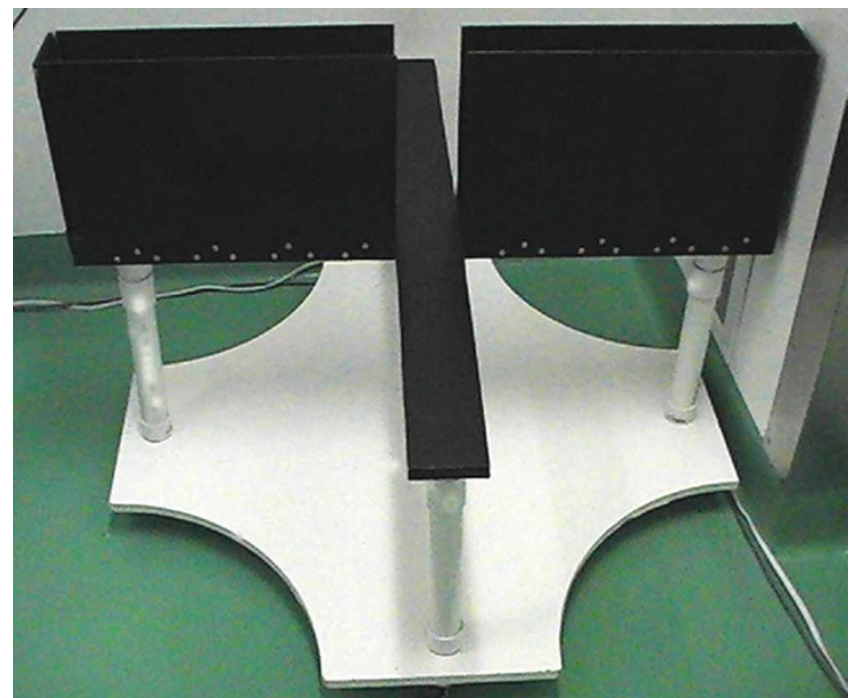

Figure 1 | Picture of the elevated plus maze used for testing rats. The elevated plus maze is placed on the floor on a movable platform with casters. Note: The elevated plus maze that we use with mice is similar, but has smaller dimensions and does not have a movable platform.

problematic, it is possible to test rodents more than once without a drop in baseline open arm exploration if there is a 3-week period between tests and the maze is moved to a novel room ${ }^{38,39}$. Indeed, it is ideal for this purpose that our rat elevated plus maze is located on a platform with wheels so that it can easily be moved to a novel room (see Fig. 1 and EQUIPMENT SETUP).

We have used single exposure to the elevated plus maze with success to determine response to novelty/anxiety behavior of rodents in different hormone conditions to determine the antianxiety effects of steroid hormones, such as estrogen, progestins and androgens $s^{22,40-50}$. The materials, methods and typical results obtained are as follows.

\section{General background information relating to the procedure}

Timing of testing: Behavior of rats and mice in the elevated plus maze can be influenced by circadian rhythms/light cycle ${ }^{14,51,52}$. In our laboratory, rats and mice, which are housed on a reverse-light cycle, are always tested in their dark phase (between 0900 and 1700 ), when rodents are most active and have consistent differences in their endogenous concentrations of corticosterone, estrogens, progestins and androgens. An important consideration to be made when setting up experiments using the elevated plus maze in each experiment is the timing of testing, and inconsistencies in what phase of the light cycle the animals are tested must be avoided to reduce potential confounding effects owing to this in behavioral analyses.

Handling of animals before testing: Prior experience with handling, stress or injections can alter behavioral responses of rodents in the elevated plus maze ${ }^{9,53-60}$. It is important to ensure that in experiments using the elevated plus maze, handling of rodents and any experience with prior stressors, particularly immediately before testing, is consistent across animals and treatment groups.

Because prior experience, as described above, can alter rodents' behavioral responses to stressor exposure and/or behavioral testing, in our laboratory, experimental animals are consistently habituated 
to being handled by experimenters, transport to the behavioral testing facility and placement in transport cages before behavioral testing. For example, in our laboratory, immediately before testing, rats and mice are placed on a cart in their homecages and pushed to the behavioral testing suite, which is down a hall (approximately 50 feet) from the housing rooms that are located within the same Laboratory Animal Care Facility. From their homecages, experimental rodents are placed separately into smaller transport cages on a cart in the hallway directly outside the behavioral testing room (there are separate rooms set up for mouse and rat elevated plus maze testing) before testing and then returned to their home cages at the end of testing. To avoid any possibility of different experience or stressor exposure altering behavior of rodents in the plus maze, it is critical that all experimental animals either spend brief, consistent time outside of their homecages in transport cages in the behavioral testing facility before testing (as is performed in our laboratory) or remain in their homecages before testing. Indeed, a basic principle of ethological behavioral analyses is to ensure that experimental animals have similar experiences and consistent treatment before behavioral analyses.

\section{MATERIALS}

\section{REAGENT SETUP}

Rats or mice Laboratory-bred rats or mice are typically used in the elevated plus maze; however, there are recent reports validating the use of the plus maze with guinea-pigs, gerbils, voles and wild mice ${ }^{61-65}$. It is particularly useful to use rats or mice in the elevated plus maze, as the equipment for these species can be easily obtained from commercial vendors and the procedures are well developed and have been validated in a number of laboratories.

Variables, related to animal subjects, that need to be considered when setting up an experiment using the elevated plus maze include: $\operatorname{strain}^{66-70}$ (which may be particularly true when considering mouse background strain when using transgenic models), breeding line differences ${ }^{71}$, sex/gender ${ }^{22,72,73}$, estrous cycle $22,74,75$ and age ${ }^{14,51,76}$.

In our laboratory, we typically use young (3-6 months old) adult male or female Long-Evans rats and mice bred on a C57 background. If female rodents are used, the stage of estrous cycle, where there are fluctuations in both estrogens (e.g., 17 $\beta$-estradiol) and progestins (e.g., progesterone), and/or hormone concentrations are considered. In our laboratory, to be able to parse out effects and reveal central mechanisms of estrogens and progestins, we often use surgical removal of the primary endogenous sources of these female-typical steroids (i.e., ovariectomy) and replacement with estrogen or progesterone. For example, administration of $17 \beta$-estradiol, which produces physiological circulating estradiol levels, increases time spent on the open arms of the plus maze of ovariectomized rodents (rats: $70 \pm 15 \mathrm{~s}$; mice: $35 \pm 9 \mathrm{~s}$ ) compared to that observed with vehicle administration (rats: $10 \pm 5 \mathrm{~s}$; mice: $5 \pm 2$ s) (see refs. 48-50,77). The results that we have obtained using our elevated plus maze protocol, with consideration of subject and procedural variables as described, are consistent across studies. Indeed, differences in these variables related to the subjects and the procedural variables need to be carefully considered when an experiment using the elevated plus maze is designed ${ }^{14}$. ! CAUTION All experiments should be performed in accordance with relevant guidelines and regulations regarding animal usage. Animal housing We obtain adult rats and mice from the breeding colony in the Life Sciences Research Buildings in the Laboratory Animal Care Facility at SUNY-Albany. The original stock of rats and mice were from Taconic Farms and Jackson Laboratory, respectively. If experimental rodents are obtained from a commercial vendor, they should be housed undisturbed for at least 1 week before testing. To avoid the effects of shipping stress on commercially purchased rodents, it is important to use at least a 1 week habituation period before initiating behavioral testing. In using a typical 1 week habituation period, we have found little evidence of differences in performance in the elevated plus maze between rodents of the same strain that are bred in-house and those purchased from vendors and shipped.

Differences in housing conditions may alter behavior in the elevated plus maze. Indeed, long-term social isolation (unlike the short-term, i.e., several minutes, isolation that experimental animals experience immediately before testing in our laboratory) is considered a stressor and a model of anxiety/ depression in mice ${ }^{78,79}$. For this reason, our rats and mice are typically grouphoused (4-5 per cage) in polycarbonate cages in a separate temperature-controlled room $\left(21 \pm 1{ }^{\circ} \mathrm{C}\right)$. Our rats and mice are maintained throughout the experiment on a $12 / 12 \mathrm{~h}$ reversed light cycle (lights off at 0800) with continuous access to Purina Rat Chow and tap water.

\section{EQUIPMENT SETUP}

Rat elevated plus maze Our maze is made of medium-density fiberboard with a matte black acrylic surface, and consists of four arms (two open without walls and two enclosed by $30 \mathrm{~cm}$ high walls) $50 \mathrm{~cm}$ long and $10 \mathrm{~cm}$ wide (Fig. 1). The open arms in the maze that we use do not have a railing, but addition of a 3-5 mm high railing on the open arms of the plus maze has been used with success to increase open arm exploration ${ }^{80,28,29,38,39}$. Each arm of the maze is attached to sturdy plastic legs, such that it is elevated $50 \mathrm{~cm}$ off a base that it is on. The legs of the maze can be easily removed to allow for storage of the maze when not in use and be adjusted so that the maze is level on uneven floors. The base consists of a white melamine platform with four casters that is constructed so that it is only as big as the maze requires. This allows for easy movement of the maze within and outside the testing room, as necessary. The maze and base were constructed within these parameters by an independent contractor, George Morimoto (gmorimoto@yahoo.com; Suffern, NY), who designed, built and set up the maze in our laboratory. We have previously used mazes from commercial vendors, such as San Diego Instruments. Other models of rat elevated plus mazes have been constructed out of other materials (i.e., plastic, stainless steel, etc.) to be collapsible for storage when not in use, but this has proven in our laboratory to produce a less sturdy construction. We do not find this to be the case with this maze built to our specifications and the data collected are comparable.

Mouse elevated plus maze Our maze is made of stainless steel, which is painted matte black, and consists of four arms (two open without walls and two enclosed by $15.25 \mathrm{~cm}$ high walls) $30 \mathrm{~cm}$ long and $5 \mathrm{~cm}$ wide. Each arm of the maze is attached to sturdy metal legs such that it is elevated $40 \mathrm{~cm}$ off of the table it is on. The maze was purchased from Columbus Instruments. In comparison to the rat elevated plus maze that we use, the mouse maze is smaller and is readily moved as necessary. As such, a mouse elevated plus maze constructed of stainless steel without a movable base is used in our laboratory. We have found similar patterns of behavior when we have used mouse elevated plus mazes from other commercial vendors and with slightly different construction (i.e., closed arms made of clear rounded Plexiglas) and when the maze was situated on the floor, instead of a table.

Video-tracking system with computer interface and video camera In our laboratory, a video-tracking system (e.g., Any-Maze, purchased from Stoelting) is used to automatically collect behavioral data. We use the program installed on a Dell PC computer with a digital video camera mounted overhead on the ceiling. Elevated plus maze location/illumination Our rat and mouse elevated plus mazes are situated in separate brightly lit rooms that are illuminated with six, 32-W fluorescent overhead lights each, which produce consistent illumination within the rooms (2,800 lumens each). The rat elevated plus maze is placed close to the center of the room, and has similar levels of illumination on both open and closed arms. The mouse elevated plus maze is placed on a table (but could also be placed on the floor) such that there are similar levels of illumination on both open and closed arms. The legs of the maze are adjusted so that the maze is perpendicular to the ground and each arm is level. Level of light can influence behavior in the elevated plus maze. As such, lighting needs to be considered when experiments using the elevated plus maze are designed.

Data collection The primary method for data collection that is used in our laboratory is the use of the video-tracking system, described above, which automatically detects and records when rats or mice enter the open or closed arms of the maze and the time spent in each. If there are problems with contrast (e.g., the rodent urinates in the maze, animal fur color, etc.), the video-tracking system is not as accurate. Although this occurs infrequently, an observer is always present in the testing room, next to the maze, and is simultaneously collecting elevated plus maze data. The observer makes hatchmarks on a data sheet for each arm entry that a rodent makes in the elevated plus maze and uses a 
timer to determine the duration spent on the open arms. This allows for comparisons between the two methods of data collection. In our laboratory, we have found that there is a greater than $95 \%$ concordance in data collected with these methods and between observers and any discordant results are due to problems with video tracking because of less than ideal contrast. As such, in the testing situation that is set up in our laboratory, it is important that the observer is in the testing room while animals are tested. However, the presence of the observer may alter rodent's behavior in this task; so this has to be considered when the task is set up in each laboratory. It is not advisable that the observer be present during testing of some experimental animals and not for others owing to the possibility of this altering the animal's behavior. Furthermore, it is critically important that the observer makes minimal movements and no noise when collecting behavioral data. In some of our testing rooms that are large enough, we have set up a curtain that hangs from the ceiling and separates the area where the elevated plus maze and observer are located, which minimizes the possibility of experimental animals reacting to the presence of the observer. However, we have found little evidence of differences in behavioral data of rodents that are tested with and without an observer that is visible to the rodent if the observer makes every effort to minimize movement and noises made.
Calibration/validation of test in laboratory Many variables can confound interpretation of behavior in the elevated plus maze, and details of how these are addressed in our own laboratory are described. Before an experiment using the elevated plus maze is performed, it is important to first calibrate the task in a particular laboratory by taking into account and minimizing these procedural and organismic variables so that the testing environment and experience is the same in each experimental animal. Use of the elevated plus maze should also be validated in each laboratory that it is set up in. This can be accomplished by using positive and negative control groups, that is, administering pharmacological anxiolytics or anxiogenics, respectively. As such, in our laboratory, we have used gonadectomized adult male rodents, which have their primary endogenous sources of androgens, such as testosterone and its metabolite (i.e., $3 \alpha$-androstanediol, a neurosteroid that is a positive modulator of $\mathrm{GABA}_{\mathrm{A}}$ receptors), the testes, removed ( $2 \pm 1 \mathrm{~s}$ spent on the open arms of the plus maze), as negative controls. Androgen-replete rodents, those that are gonadally intact $(28 \pm 6 \mathrm{~s})$ or gonadectomized and replaced with testosterone $\left(4 \mathrm{mg} \mathrm{kg}^{-1}\right.$ subcutaneously; $36+5 \mathrm{~s}$, which produces physiological circulating testosterone and $3 \alpha$-androstanediol levels) serve as positive controls ${ }^{40,41}$.

\section{PROCEDURE}

1) Make sure maze is cleaned and dried before use and that video-tracking system is ready to be used. Fill out data sheets with subject number of animal, date, coded condition and initialed by the experimenter before testing.

2| Bring rodent, which is in its individual temporary transport cage, into the behavioral testing room.

3| Take rodent out of its cage and place at the junction of the open and closed arms, facing the open arm opposite to where the experimenter is.

$\triangle$ CRITICAL STEP Make sure to handle the rodent in a consistent manner and place each rodent in the elevated plus maze in the same position. Differences are observed when rodents are placed facing toward an open arm versus toward the closed arm ${ }^{1}$. In our laboratory, rodents are placed on the maze facing the same open arm.

4 Start the video-tracking system and a timer set for 5 min when the rodent is placed in the maze.

$\triangle$ CRITICAL STEP Take precautions to start data collection as soon as the animal is placed in the maze. It is not recommended that the video-tracking system is started before the animal is placed in the maze so that the behavior of each animal is consistently recorded for $5 \mathrm{~min}$.

5| The video-tracking system will automatically record the number of entries made by the rodent onto the open and closed arms and the time spent on the open arm and closed arm. The experimenter should simultaneously record the number of arm entries and time spent in each open arm on the data sheets with a timer.

$\triangle$ CRITICAL STEP An open arm entry is counted when all four paws of the rodent are on the open arm.

$\triangle$ CRITICAL STEP The observer must avoid unnecessary movements and making noise.

6| At the end of the 5-min test, remove the rodent from the plus maze and place into a transport cage. Place back inside its homecage on the cart outside the room.

7| Clean the elevated plus maze with quatricide and dry with paper towels before testing with another rodent.

\section{Data analyses}

8| To determine the effects of experimental manipulation (i.e., steroid hormones in our laboratory), differences in the raw means of experimental animals should be typically calculated with analyses of variance. We use a commercially available statistical analyses program, such as Statview, but freeware statistics programs could also be used. When main effects of manipulation/independent variables are revealed, using a $P$-value of less than 0.05 , the data can be further analyzed with post hoc tests, such as Tukey's HSD, which corrects for multiple comparisons, to determine differences between groups.

Typically, analysis of variance can be used to assess the effects of treatment on the following measures: open arm time, closed arm time, open arm entries made, closed arm entries made and total entries made. The ratio of open or closed arm entries/time to the total arm entries/time can be calculated and analyzed. This may be particularly helpful if there are differences between groups in the general motor activity in the maze. Other ethological measures (which can be considered "risk assessment") that can be collected are the frequency and/or duration of head dips (downward movement of rodents' head toward the floor from the open arms), rears (vertical standing of rodent on two hindlegs) and stretch-attend posture (when the rodent is motionless, but has body stretched forward/toward a stimulus). 
PROTOCOL

\section{TIMING}

Steps 1 and 2 (setup): $0.5-1 \mathrm{~h}$ for cohort of experimental animals

Steps 3-5 (testing): 5 min per animal

Steps 6 and 7 (clean-up): 1-2 min per animal

\section{? TROUBLESHOOTING}

\section{Rodents fall off open arms}

Infrequently (in less than $1 \%$ of the rodents tested), rats or mice run to the edge of the open arms and fall off. When this occurs, the experimenter must rapidly pick the animal up and place it back onto the open arms of the maze. This behavior must be recorded on the data sheet and taken into consideration when the behavioral data are analyzed. Behavioral data from an animal that does this would be excluded from analyses. However, the experimenter continues to test the animal because it is important to make sure that exposure to the elevated plus maze is as consistent across animals as possible, particularly when animals are tested in a battery of tasks.

\section{Rodents are immobile/freeze on open arms}

If a noise or movement occurs while rodents are on the open arms of the elevated plus maze, rodents are likely to freeze for an extended period of time, even the majority of the testing time, on the open arms of the maze. Freezing for an extended period of time would be considered when the time spent on the open arms is more than $30 \%$ of the total test time (i.e., more than $100 \mathrm{~s}$ ). This is a rare occurrence (i.e., occurs in less than $1 \%$ of the animals that are tested).

If freezing were to occur, the experimenter must make a note of this on their data sheets and consider this anomaly when analyzing the behavioral results. If a loud noise or other disruption occurs, which are considered exclusionary criteria, the animals' data are not considered in data analyses. However, the experimenter continues to test the animal so that it is exposed to the elevated plus maze for 5 min (to be commensurate with other animals in its cohort). To avoid increased freezing of the rodent to occur in the plus maze, noise and movements made by the experimenter must be kept at a minimum. In our laboratory, we hang signs outside the testing rooms to inform others that testing is in progress so that noise and disruptions are kept at a minimum.

\section{Different baseline open arm activity}

It is critical to decide upon the sex and reproductive age of animals to be used before initiating a study using the elevated plus maze. Post-pubertal sex difference in the elevated plus maze has been reported 22,73 . Historically, many studies have used male rodents to avoid the influence of estrous cycle for elevated plus maze behavior ${ }^{22,74,75}$. For example, in our laboratory, we have consistently observed that young, adult intact female rats or mice in diestrus (low estradiol, low progestins) spent less time (rats: $20 \pm 3$ s, mice: $6 \pm 2 \mathrm{~s}$ ) than do their intact male counterparts (rats: $35 \pm 5 \mathrm{~s}$; mice: $21 \pm 6 \mathrm{~s}$ ) in the open arms. However, the pattern of elevated plus maze behavior is different when rodents in proestrous (high estradiol and progestins) are tested; rodents in proestrous (rats: $75 \pm 25 \mathrm{~s}$; mice: $30 \pm 5 \mathrm{~s}$ ) spend more time on the open arms of the elevated plus maze than do rodents in diestrous or males. Therefore, investigators must take into consideration that there may be different baseline elevated plus maze activity depending on the sex of experimental animals and estrous cycle stage in females, and design experiments using the elevated plus maze accordingly.

\section{ANTICIPATED RESULTS}

The following results exemplify data we have obtained using the elevated plus maze protocol, which is described in detail above, to investigate the anti-anxiety effects of progesterone ${ }^{43,46}$. The results we have obtained using the elevated plus maze to determine the effects of progesterone and its metabolites are robust and replicable. Furthermore, similar to androgens, described above, progesterone is metabolized to a neuroactive steroid, called allopregnanolone, that positively modulates $\mathrm{GABA}_{\mathrm{A}}$ receptors and produces similar anxiolytic effects as benzodiazepines ${ }^{44}$. Indeed, there is a good correlation between typical behavioral effects of progestins in the elevated plus maze with the other anxiety/affective measures that use exposure to bright, open spaces like the elevated plus maze or other noxious stimuli (i.e., electric shock, food/water deprivation, loud noises, predator odor and forced swim/confinement) that we use in the laboratory, such as the open field, elevated zero maze, mirrored chamber task, light-dark transition task, vogel punished drinking, defensive freezing task, conditioned fear, predator stress-induced analgesia, porsolt forced swimming task and tail suspension task. An experiment conducted in our laboratory using the elevated plus maze is described below.

In this study, young adult (2-3 months old) female rats and mice were ovariectomized and recovered from surgery for 1 week. One hour before testing in the elevated plus maze, rats or mice were administered progesterone $\left(4 \mathrm{mg} \mathrm{kg}^{-1}\right.$, rats, or $1 \mathrm{mg}$, mice, which produces similar plasma and central levels of progestins as are observed in proestrous rodents) via subcutaneous injections. An observer, blind to the experimental condition of the experimental animal, recorded the behavioral data using the protocol described in detail above. Data were analyzed with analyses of variance, followed by Tukey's HSD post hoc tests, as appropriate (i.e., when $P$-value of main effect of progesterone condition was less than 0.05 ). We found that administration of 
progesterone increases time spent on the open arms of the plus maze in rats $(65 \pm 9 \mathrm{~s})$ and mice $(21 \pm 8 \mathrm{~s})$ compared to vehicle administration (rats: $9 \pm 5 \mathrm{~s}$; mice: $4 \pm 2 \mathrm{~s}$ ). Thus, we can conclude that progesterone can increase anti-anxiety-like behavior in the elevated plus maze.

\section{Summary}

The elevated plus maze is a widely used behavioral assay for anxiety behavior of rodents. It is easy to use, can be fully automated and valid results can be obtained in a short, 5-min testing period. The patterns of results obtained using this task are replicable across other species, anxiety/affective behavior measures, studies and laboratories.

ACKNOWLEDGMENTS This research was supported by a grant from the National Institute of Mental Health (MH06769801), National Science Foundation (IBN03-16083) and US Army Department of Defense (BC051001).

COMPETING INTERESTS STATEMENT The authors declare that they have no competing financial interests.

Published online at http://www.natureprotocols.com

Reprints and permissions information is available online at http://npg.nature.com/ reprintsandpermissions

1. Pellow, S., Chopin, P., File, S.E. \& Briley, M. Validation of open:closed arm entries in an elevated plus-maze as a measure of anxiety in the rat. J. Neurosci. Methods 14, 149-167 (1985).

2. Montgomery, K.C. The relation between fear induced by novel stimulation and exploratory behavior. J. Comp. Physiol. Psychol. 48, 254-260 (1958).

3. Handley, S.L. \& Mithani, S. Effects of $\alpha$-adrenoreceptor agonists and antagonists in a maze-exploration model of 'fear'-motivated behaviour. NaunynSchmeideberg's Arch. Pharmacol. 327, 1-5 (1984).

4. Barnett, SA. The Rat-A Study in Behavior. (Univ. Chicago Press, Chicago, 1975).

5. Gonzalez, L.E. \& File, S.E. A five minute experience in the elevated plus-maze alters the state of the benzodiazepine receptor in the dorsal raphe nucleus. J. Neurosci. 17, 1505-1511 (1997).

6. Silveira, M.C., Sandner, G. \& Graeff, F.G. Induction of Fos immunoreactivity in the brain by exposure to the elevated plus-maze. Behav. Brain Res. 56, 115-118 (1993).

7. Cortese, B.M. \& Phan, K.L. The role of glutamate in anxiety and related disorders. CNS Spectr. 10, 820-830 (2005).

8. Lister, R.G. The use of a plus-maze to measure anxiety in the mouse. Psychopharmacology 92, 180-185 (1987).

9. Mechiel Korte, S. \& De Boer, S.F. A robust animal model of state anxiety: fear-potentiated behaviour in the elevated plus-maze. Eur. J. Pharmacol. 463, 163-175 (2003).

10. Overstreet, D.H., Commissaris, R.C., De La Garza, R. 2nd, File, S.E., Knapp, D.J. \& Seiden, L.S. Involvement of 5-HT1A receptors in animal tests of anxiety and depression: evidence from genetic models. Stress 6 , 101-110 (2003).

11. Rodgers, R.J., Lee, C. \& Shepherd, J.K. Effects of diazepam on behavioural and antinociceptive responses to the elevated plus-maze in male mice depend upon treatment regimen and prior maze experience. Psychopharmacology (Berl.) 106, 102-110 (1992).

12. Silva, R.C. \& Brandao, M.L. Acute and chronic effects of gepirone and fluoxetine in rats tested in the elevated plus-maze: an ethological analysis. Pharmacol. Biochem. Behav. 65, 209-216 (2000).

13. Belzung, C. \& Griebel, G. Measuring normal and pathological anxiety-like behaviour in mice: a review. Behav. Brain Res. 125, 141-149 (2001).

14. Carobrez, A.P. \& Bertoglio, L.J. Ethological and temporal analyses of anxiety-like behavior: the elevated plus-maze model 20 years on. Neurosci. Biobehav. Rev. 29, 1193-1205 (2005).

15. Dawson, G.R. \& Tricklebank, M.D. Use of the elevated plus maze in the search for novel anxiolytic agents. Trends Pharmacol. Sci. 16, 33-36 (1995).

16. File, S.E. Factors controlling measures of anxiety and responses to novelty in the mouse. Behav. Brain Res. 125, 151-157 (2001).

17. Hogg, S. A review of the validity and variability of the elevated plus-maze as an animal model of anxiety. Pharmacol. Biochem. Behav. 54, 21-30 (1996).

18. Kulkarni, S.K. \& Sharma, A.C. Elevated plus-maze: a novel psychobehavioral tool to measure anxiety in rodents. Methods Find. Exp. Clin. Pharmacol. 13, 573-577 (1991).

19. Rodgers, R.J. \& Dalvi, A. Anxiety, defence and the elevated plus-maze. Neurosci. Biobehav. Rev. 21, 801-810 (1997).
20. Wahlsten, D. et al. Different data from different labs: lessons from studies of gene-environment interaction. J Neurobiol. 54, 283-311 (2003).

21. Wall, P.M. \& Messier, C. Methodological and conceptual issues in the use of the elevated plus-maze as a psychological measurement instrument of animal anxiety-like behavior. Neurosci. Biobehav. Rev. 25, 275-286 (2001)

22. Frye, C.A., Petralia, S.M. \& Rhodes, M.E. Estrous cycle and sex differences in performance on anxiety tasks coincide with increases in hippocampal progesterone and $3 \alpha, 5 \alpha-$ THP. Pharmacol. Biochem. Behav. 67, 587-596 (2000).

23. File, S.E., Zangrossi, H. Jr, Sanders, F.L. \& Mabbutt, P.S. Raised corticosterone in the rat after exposure to the elevated plus-maze. Psychopharmacology 113 543-546 (1994).

24. Rodgers, R.J., Haller, J., Holmes, A., Halasz, J., Walton, T.J. \& Brain, P.F. Corticosterone response to the plus-maze: high correlation with risk assessment in rats and mice. Physiol. Behav. 68, 47-53 (1999).

25. File, S.E. \& Wardill, A.G. Validity of head-dipping as a measure of exploration in a modified hole-board. Psychopharmacologia 44, 53-59 (1975).

26. File, S.E. \& Wardill, A.G. The reliability of the hole-board apparatus. Psychopharmacologia 44, 47-51 (1975).

27. Weiss, S.M., Wadsworth, G., Fletcher, A. \& Dourish, C.T. Utility of ethological analysis to overcome locomotor confounds in elevated maze models of anxiety. Neurosci. Biobehav. Rev. 23, 265-271 (1998).

28. Adamec, R., Strasser, K., Blundell, J., Burton, P. \& McKay, D.W. Protein synthesis and the mechanisms of lasting change in anxiety induced by severe stress. Behav. Brain Res. 167, 270-286 (2006).

29. Adamec, R., Blundell, J. \& Burton, P. Anxiolytic effects of kindling role of anatomical location of the kindling electrode in response to kindling of the right basolateral amygdala. Brain Res. 1024, 44-45 (2004).

30. File, S.E., Mabbutt, P.S. \& Hitchcott, P.K. Characterisation of the phenomenon of "one-trial tolerance" to the anxiolytic effect of chlordiazepoxide in the elevated plus-maze. Psychopharmacology 102, 98-101 (1990).

31. Almeida, S.S., Garcia, R.A. \& de Oliveira, L.M. Effects of early protein malnutrition and repeated testing upon locomotor and exploratory behaviors in the elevated plus-maze. Physiol. Behav. 54, 749-752 (1993).

32. Bertoglio, L.J. \& Carobrez, A.P. Previous maze experience required to increase open arms avoidance in rats submitted to the elevated plus-maze model of anxiety. Behav. Brain Res. 108, 197-203 (2000).

33. Bertoglio, L.J. \& Carobrez, A.P. Anxiolytic effects of ethanol and phenobarbital are abolished in test-experienced rats submitted to the elevated plus maze. Pharmacol. Biochem. Behav. 73, 963-969 (2002).

34. Bertoglio, L.J. \& Carobrez, A.P. Behavioral profile of rats submitted to session 1-session 2 in the elevated plus-maze during diurnal/nocturnal phases and under different illumination conditions. Behav. Brain Res. 132, 135-143 (2002).

35. Fernandes, C. \& File, S.E. The influence of open arm ledges and maze experience in the elevated plus-maze. Pharmacol. Biochem. Behav. 54, 31-40 (1996).

36. Lee, C. \& Rodgers, R.J. Antinociceptive effects of elevated plus-maze exposure: influence of opiate receptor manipulations. Psychopharmacology 102, 507-513 (1990).

37. Treit, D., Menard, J. \& Royan, C. Anxiogenic stimuli in the elevated plus-maze. Pharmacol. Biochem. Behav. 44, 463-469 (1993).

38. Adamec, R. \& Shallow, T. Effects of baseline anxiety on response to kindling of the right medial amygdala. Physiol. Behav. 70, 67-80 (2000).

39. Adamec, R., Shallow, T. \& Burton, P. Anxiolytic and anxiogenic effects of kindling - role of baseline anxiety and anatomical location of the kindling electrode in response to kindling of the right and left basolateral amygdala. Behav. Brain Res. 159, 73-88 (2005).

40. Edinger, K.L. \& Frye, C.A. Testosterone's anti-anxiety and analgesic effects may be due in part to actions of its 5alpha-reduced metabolites in the hippocampus. Psychoneuroendocrinology 30, 418-430 (2005). 
41. Frye, C.A. \& Lacey, E.H. Posttraining androgens' enhancement of cognitive performance is temporally distinct from androgens' increases in affective behavior. Cogn. Affect. Behav. Neurosci. 1, 172-182 (2001).

42. Frye, C.A. \& Seliga, A.M. Testosterone increases analgesia, anxiolysis, and cognitive performance of male rats. Cogn. Affect. Behav. Neurosci. 1, 371-381 (2001).

43. Frye, C.A. \& Rhodes, M.E. Infusions of $5 \alpha$-pregnan-3 $\alpha$-ol-20-one $(3 \alpha, 5 \alpha$-THP) to the ventral tegmental area, but not the substantia nigra, enhance exploratory, anti-anxiety, social and sexual behaviours and concomitantly increase $3 \alpha, 5 \alpha-$ THP concentrations in the hippocampus, diencephalon and cortex of ovariectomised oestrogen-primed rats. J. Neuroendocrinol. 18, 960-975 (2006).

44. Frye, C.A., Rhodes, M.E., Petralia, S.M., Walf, A.A., Sumida, K. \& Edinger, K.L. $3 \alpha$-Hydroxy-5 $\alpha$-pregnan-20-one in the midbrain ventral tegmental area mediates social, sexual, and affective behaviors. Neuroscience 138, 1007-1014 (2006).

45. Frye, C.A., Walf, A.A., Rhodes, M.E. \& Harney, J.P. Progesterone enhances motor, anxiolytic, analgesic, and antidepressive behavior of wild-type mice, but not those deficient in type 15 alpha-reductase. Brain Res. 1004, 116-124 (2004).

46. Frye, C.A. \& Walf, A.A. Estrogen and/or progesterone systemically or to the amygdala can have anxiety, fear, and pain reducing effects in ovx rats. Behav. Neurosci. 118, 306-313 (2004).

47. Rhodes, M.E. \& Frye, C.A. Inhibiting progesterone metabolism in the hippocampus of rats in behavioral estrus decreases anxiolytic behaviors and enhances exploratory and antinociceptive behaviors. Cogn. Affect. Behav. Neurosci. 1, 287-296 (2001).

48. Walf, A.A. \& Frye, C.A. Estradiol's effects to reduce anxiety and depressive behavior may be mediated by estradiol dose and restraint stress. Neuropsychopharmacology 30, 1288-1301 (2005).

49. Walf, A.A. \& Frye, C.A. ER $\beta$-selective estrogen receptor modulators produce antianxiety behavior when administered systemically to ovariectomized rats. Neuropsychopharmacology 30, 1598-1609 (2005).

50. Walf, A.A. \& Frye, C.A. A review and update of: mechanisms of estrogen in the hippocampus and amygdala for anxiety and depression behavior. Neuropsychopharmacology 31, 1097-1111 (2006).

51. Andrade, M.M., Tome, M.F., Santiago, E.S., Lucia-Santos, A. \& de Andrade, T.G. Longitudinal study of daily variation of rats' behavior in the elevated plus-maze. Physiol. Behav. 78, 125-133 (2003).

52. Jones, N. \& King, S.M. Influence of circadian phase and test illumination on preclinical models of anxiety. Physiol. Behav. 72, 99-106 (2001).

53. Adamec, R.E. \& Shallow, T. Lasting effects on rodent anxiety of a single exposure to a cat. Physiol. Behav. 54, 101-109 (1993).

54. Andrews, N. \& File, S.E. Handling history of rats modifies behavioural effects of drugs in the elevated plus-maze test of anxiety. Eur. J. Pharmacol. 235, 109-112 (1993).

55. Brett, R.R. \& Pratt, J.A. Chronic handling modifies the anxiolytic effect of diazepam in the elevated plus-maze. Eur. J. Pharmacol. 178, 135-138 (1990).

56. File, S.E., Andrews, N., Wu, P.Y., Zharkovsky, A. \& Zangrossi, H. Jr. Modification of chlordiazepoxide's behavioural and neurochemical effects by handling and plus-maze experience. Eur. J. Pharmacol. 218, 9-14 (1992).

57. Lapin, I.P. Only controls: effect of handling, sham injection, and intraperitoneal injection of saline on behavior of mice in an elevated plus-maze. J. Pharmacol. Toxicol. Methods 34, 73-77 (1995).

58. Padovan, C.M. \& Guimaraes, F.S. Restraint-induced hypoactivity in an elevated plus-maze. Braz. J. Med. Biol. Res. 33, 79-83 (2000).

59. Schmitt, U. \& Hiemke, C. Strain differences in open-field and elevated plus-maze behavior of rats without and with pretest handling. Pharmacol. Biochem. Behav. 59, 807-811 (1998).
60. Steenbergen, H.L., Heinsbroek, R.P., Van Hest, A. \& Van de Poll, N.E. Sexdependent effects of inescapable shock administration on shuttlebox-escape performance and elevated plus-maze behavior. Physiol. Behav. 48, 571-576 (1990).

61. Hendrie, C.A., Eilam, D. \& Weiss, S.M. Effects of diazepam and buspirone on the behaviour of wild voles (Microtus socialis) in two models of anxiety. Pharmacol. Biochem. Behav. 58, 573-576 (1997).

62. Holmes, A., Parmigiani, S., Ferrari, P.F., Palanza, P. \& Rodgers, R.J. Behavioral profile of wild mice in the elevated plus-maze test for anxiety. Physiol. Behav. 71, 509-516 (2000).

63. Rex, A., Marsden, C.A. \& Fink, H. Effect of diazepam on cortical 5-HT release and behaviour in the guinea-pig on exposure to the elevated plus maze. Psychopharmacology 110, 490-496 (1993).

64. Stowe, J.R., Liu, Y., Curtis, J.T., Freeman, M.E. \& Wang, Z. Species differences in anxiety-related responses in male prairie and meadow voles: the effects of social isolation. Physiol. Behav. 86, 369-378 (2005).

65. Varty, G.B., Morgan, C.A., Cohen-Williams, M.E., Coffin, V.L. \& Carey, G.J. The gerbil elevated plus-maze I: behavioral characterization and pharmacological validation. Neuropsychopharmacology 27, 357-370 (2002).

66. Carola, V., D'0limpio, F., Brunamonti, E., Mangia, F. \& Renzi, P. Evaluation of the elevated plus-maze and open-field tests for the assessment of anxiety-related behaviour in inbred mice. Behav. Brain Res. 134, 49-57 (2002).

67. Ramos, A., Berton, 0., Mormede, P. \& Chaouloff, F. A multiple-test study of anxiety-related behaviours in six inbred rat strains. Behav. Brain Res. $\mathbf{8 5}$ 57-69 (1997).

68. Rodgers, R.J. \& Cole, J.C. Influence of social isolation, gender, strain, and prior novelty on plus-maze behaviour in mice. Physiol. Behav. 54, 729-736 (1993).

69. Trullas, R. \& Skolnick, P. Differences in fear motivated behaviors among inbred mouse strains. Psychopharmacology 111, 323-331 (1993).

70. Voikar, V., Koks, S., Vasar, E. \& Rauvala, H. Strain and gender differences in the behavior of mouse lines commonly used in transgenic studies. Physiol. Behav. $\mathbf{7 2}$ 271-281 (2001).

71. Bert, B., Fink, H., Sohr, R. \& Rex, A. Different effects of diazepam in Fischer rats and two stocks of Wistar rats in tests of anxiety. Pharmacol. Biochem. Behav. 70, 411-420 (2001).

72. Imhof, J.T., Coelho, Z.M., Schmitt, M.L., Morato, G.S. \& Carobrez, A.P. Influence of gender and age on performance of rats in the elevated plus maze apparatus. Behav. Brain Res. 56, 177-180 (1993).

73. Johnston, A.L. \& File, S.E. Sex differences in animal tests of anxiety. Physiol. Behav. 49, 245-250 (1991).

74. Marcondes, F.K., Miguel, K.J., Melo, L.L. \& Spadari-Bratfisch,, R.C. Estrous cycle influences the response of female rats in the elevated plus-maze test. Physiol. Behav. 74, 435-440 (2001).

75. Mora, S., Dussaubat, N. \& Diaz-Veliz, G. Effects of the estrous cycle and ovarian hormones on behavioral indices of anxiety in female rats. Psychoneuroendocrinology 21, 609-620 (1996).

76. Boguszewski, P. \& Zagrodzka, J. Emotional changes related to age in rats-a behavioral analysis. Behav. Brain Res. 133, 323-332 (2002).

77. Nomikos, G.G. \& Spyraki, C. Influence of oestrogen on spontaneous and diazepam-induced exploration of rats in an elevated plus maze. Neuropharmacology 27, 691-696 (1988).

78. Hunt, C. \& Hambly, C. Faecal corticosterone concentrations indicate that separately housed male mice are not more stressed than group housed males. Physiol. Behav. 87, 519-526 (2006).

79. Zhu, S.W., Yee, B.K., Nyffeler, M., Winblad, B., Feldon, J. \& Mohammed, A.H. Influence of differential housing on emotional behaviour and neurotrophin levels in mice. Behav. Brain Res. 169, 10-20 (2006).

80. Treit, D.J., Menard \& Royan, C. Anxiogenic stimuli in the elevated plus-maze. Pharmacol. Biochem. Behav. 44, 463-469 (1993). 